\title{
Different Modalities of Breast Reconstruction with Autologous Latissimus Dorsi Flap: Aesthetic Results and Complications
}

\author{
Hussein Fakhry ${ }^{1, *}$, Kassim Abdelazeem ${ }^{1}$, Hesham Hamza ${ }^{1}$, Badawy Ahmed $^{1}$, Mahmod Mostafa ${ }^{1}$, \\ Gamal Amira ${ }^{2}$, Arwa M. Ali ${ }^{3}$
}

${ }^{1}$ Surgical Oncology Department, South Egypt Cancer Institute, Assiut University, Assiut, Egypt

${ }^{2}$ Surgical Oncology Department, National Cancer Institute, Cairo University, Assiut, Egypt

${ }^{3}$ Medical Oncology Department, South Egypt Cancer Institute, Assiut University, Assiut, Egypt

Email address:

Kassimabdelazeem@yahoo.com (K. Abdelazeem)

\section{To cite this article:}

Hussein Fakhry, Kassim Abdelazeem, Hesham Hamza, Badawy Ahmed, Mahmod Mostafa, Gamal Amira, Arwa M. Ali. Different Modalities of Breast Reconstruction with Autologous Latissimus Dorsi Flap: Aesthetic Results and Complications. Journal of Surgery.

Vol. 3, No. 5, 2015, pp. 44-49. doi: 10.11648/j.js.20150305.11

\begin{abstract}
Background. Use of an autologous latissimus dorsi (LD) flap in breast reconstruction accounts for a flexible and natural look of reconstructed breast and has maintained a strong popularity because of its ease of harvest, reliability, and ability to provide additional prosthetic coverage. Different complications (hematoma, seroma, flap necrosis, infection, hypertrophic scarring, and postoperative back pain) linked to this type of breast reconstruction. The aim of this study was to evaluate the complications and aesthetic outcome of (LD) flap breast reconstruction after breast cancer surgery. Materials and methods. forty patients underwent breast reconstruction using (LD) flap with a follow-up period ranged from 6 to 18 months. Patients with small to medium sized breasts underwent complete reconstruction by extended (LD) flap after mastectomy either subcutaneous or skin sparing mastectomy while patients with large pendulous breast underwent Augmentation by (LD) miniflap after conservative surgery by wide local excision (WLE) with safety margin. All patients gave their informed consent for the procedure and were aware of the potential complications and the possibility of secondary procedures. Results. The ages of the patients in our study ranged from 25 to 65 years old. 28 (70\%) patients underwent (WLE) and reconstruction with (LD) miniflap while modified radical mastectomy, skin sparing mastectomy and subcutaneous mastectomy were done in $2(5 \%)$, $4(10 \%)$ and $6(15 \%)$ patients respectively with complete reconstruction by extended(LD) flap. The complication rates were noted as follows: partial flap necrosis in 4 patients (10\%), wound breakdown in 2 patients $(5 \%)$, lymphorrhea in 2 patients $(5 \%)$, seroma in 6 patients $(15 \%)$. Some of patients showed a minor deformity in the back, which disappeared with time and most patients, had temporary limitation of shoulder movements postoperatively but all recovered completely within few weeks. No patients underwent secondary nipple and areola reconstruction. No local recurrence or distant metastasis in any patient during the follow up period of our study. Evaluation of aesthetic results by patients revealed that 30 patients $(75 \%)$ were deeply satisfied, 6 patients $(15 \%)$ were satisfied and 4 patients $(10 \%)$ were poorly satisfied. While, surgeon aesthetic evaluation was good in 28 patients $(70 \%)$, satisfactory in 8 patients $(20 \%)$ and fair in 4 patients $(10 \%)$. Conclusion. (LD) flap breast reconstruction is a very versatile, safe and satisfactory technique with high success rate and is even suitable for high-risk patients. Donor site seroma is the most common complication and can be treated by repeated aspiration in outpatient clinic. Latissimus dorsi (LD) miniflap is the mainstay of breast reconstruction after partial mastectomy to repair defects in the lateral quadrants and the lower inner pole with low donor site morbidity and deep patient satisfaction.
\end{abstract}

Keywords: Breast Cancer, Latissimus Dorsi Flap, Breast Reconstruction, Complications

\section{Introduction}

Mastectomy was the standard treatment of breast cancer until late of the 20th century. Then breast-conservation surgery (BCS) is established as a safe option for most women with early breast cancer [1]. In fact, approximately $10 \%$ to $30 \%$ of patients submitted to BCS are not satisfied with the 
aesthetic outcome due to main reasons related to the tumour resection, which can produce asymmetry, retraction and volume changes in the breast. In addition, radiation can also have a negative effect on the native breast [2].

Recently, more attention has been focused on oncoplastic procedures In fact, the modern oncoplastic breast surgery combines principles of oncologic, plastic surgery techniques to obtain oncologically sound, and aesthetically pleasing results. Thus, by means of customized techniques the surgeon ensures that oncologic principles are not jeopardized while meeting the needs of the patient from an aesthetic point of view and psychologic problems [3]

Breast reconstruction is nowadays an integral part of rehabilitation after mastectomy. Surgical planning and timing of reconstruction should include breast volume, tumor location, the extent of glandular tissue resected, enabling each patient to receive an individualized "custom-made" reconstruction. Either immediately after resection (primary reconstruction) or later, usually after completed oncologic treatment (secondary reconstruction) [4]. Immediate breast reconstruction is gaining wide support due to its evident benefits-single stage procedure, better psychological adjustment and better esthetic outcome and easier breast reshaping [5].

The application of oncoplastic surgery depends on the tumor size, tumor location, ratio of tumor to whole breast volume, and range of excision volume. The latissimus dorsi myocutaneous flap (LDMCF) is a useful method as oncoplastic breast surgery. LDMCF can supply adequate volume and be easily acquired. Besides, it has an advantage of low complication rates with adequate safe blood supply, relatively simple surgical technique and easy closure of the donor region $[5,6]$.

Of the various autologous tissue reconstructions for postexcision defects in conserved breasts, the latissimus dorsi miniflap (LDMF) is steadily gaining popularity among nonplastic breast surgeons. The LDMF replenishes loss of more than $25 \%$ of breast volume allowing conservation of most of the natural breast tissue that otherwise would have been impossible from an oncological standpoint $[7,8]$

Our aim of this prospective study was to evaluate the complications and aesthetic outcome of (LDF) breast reconstruction after breast cancer surgery.

\section{Patients and Methods}

Immediate breast reconstruction (IBR) using (LD) flap were performed for 40 patients with small to medium sized breasts underwent complete reconstruction by extended (LD) flap after mastectomy either subcutaneous or skin sparing mastectomy while patients with large pendulous breast underwent augmentation. In the period from January 2010 to January 2012 with a follow-up period ranged from six to eighteen months, all patients were operated upon at Surgical Oncology Department, South Egypt Cancer Institute.

All patients were diagnosed and underwent routine laboratory investigations including complete blood count, liver and kidney functions as well as chest $\mathrm{X}$ ray. The staging process based on carrying out routine digital mammography complemented with an ultrasound examination to both breasts and the axilla, bone scan and CT CAB as per NCCN guidelines recommendation. Histopathological examination performed through a free hand core biopsy for superficial tumour and an ultrasound guided biopsy for deeply seated tumour. The exclusions criteria were (large or central tumors in small breasts, multifocal/multicentric disease, an extensive in situ component and widespread lymphatic invasion.

The decision for IBR was based on comprehensive preoperative information and advice that provided in a multidisciplinary setting. An informed consent was obtained from each patient after discussing the details of the operation as well as the possible intraoperative and postoperative sequel.

\section{Surgical Technique}

The Latissimus Dorsi Myocutaneous Flap (LDMCF) was used to reconstruct the resection defects, replacing the volume excised with autogenous tissue. The affected quadrant was accessed then the tumor was gripped digitally and a (WLE) with a minimum of $2 \mathrm{~cm}$ away from the palpable margin of the tumor was carried out. The specimen thus included tumor with surrounding normal breast tissue, subcutaneous fat and pectoralis fascia.

Axillary dissection was adequately performed through the superolateral end of the incision or through a separate incision. We used the transversely oriented incision for our patients and LDMF was raised with the patient in the lateral decubitus position with a $90^{\circ}$ abducted shoulder. Preoperatively the bra strap area and the inframammary crease were marked out with the patient standing.

When skin was needed, the transverse skin paddle was marked on the back by the pinch technique along the desired line. The incision went down to the subdermal layer. The plane of dissection then continued along the subcutaneous plane just above Scarpa's fascia leaving at least one $\mathrm{cm}$-thick native skin flaps. The thoracodorsal vessels are kept intact during axillary dissection. Its anterior border was then separated carefully from the underlying serratus anterior muscle. The insertion of the muscle into the intertubercle groove on the humerus was either totally or in some cases subtotally divided to keep the pedicle protected and at the same time to minimizes the axillary bulk, which would be caused if whole insertion were to be left. The thoracodorsal nerve was divided to avoid contraction of the muscle.

The patients and two surgeons assessed the aesthetic results independently. The assessment by surgeons was determined by evaluating the preoperative and postoperative photographs for breast shape and contour, definition of the inframammary fold and the anterior axillary line, the creation of inferior fullness, the degree of symmetry to the other breast and the quality of the scars. The patients' aesthetic 
evaluation was based on their subjective satisfaction with the shape of the new breast, the degree of symmetry to the contralateral breast, its consistency and the quality of the scars.

The aesthetic results have been ranked into three categories by the surgeons: good, satisfactory and fair and satisfaction of patients has been classified into three levels: deeply satisfied, satisfied and poorly satisfied. Postoperative complications and their management and secondary operations required were also recorded.

\section{Neoadjuvant and Adjuvant Chemotherapy}

Eligible patients (14 patients) received adjuvant chemotherapy. Chemotherapy regimen was six cycles of Anthracycline-based chemotherapy (FAC). FAC (5fluorouracil $500 \mathrm{mg} / \mathrm{m} 2$, Adriamycin $50 \mathrm{mg} / \mathrm{m} 2$, and cyclophosphamide $500 \mathrm{mg} / \mathrm{m} 2$ ) every 3 weeks for total of 6 cycles epirubcin $100 \mathrm{mg} / \mathrm{m}^{2}$ replaced adriamycine in case of cardio toxicity risk factors.

In cases of T3 tumor or clinically palpable axillary lymph nodes patients and those with positive axillary lymph node by axillary ultrasound examination (14 patients) had received (FAC) as a neoadjuvant chemotherapy. All 6 cycles are given before surgery with interval radiologic assessment to confirm the response. Tumors, which did not respond after 3 cycle of FAC, received alternate chemotherapy regimen in the form of Docitaxel $100 \mathrm{mg} / \mathrm{m}^{2}$ every 3 weeks.

Premenopausal patients with hormonal receptor responsive tumors received adjuvant hormonal treatment in the form of tamoxifen $20 \mathrm{mg}$ daily. Postmenopausal hormonal responsive tumors received AI ( letrazole) $2.5 \mathrm{mg}$ PO once daily. All patients were under regular follow up until the end of the study.

\section{Results}

From January 2010 till January 2012, forty female patients whom were selected from outpatient's clinic of surgical oncology department underwent breast reconstruction using (LDF). Immediate reconstruction was done for all patients. The age of the patients in our study range from 25 to 65 years with a follow-up period ranging from 6 to 18 months. Body mass index range was 21-33 kg/m2 (mean 27); operative time was 2.5-3.5 hours (mean $3 \mathrm{hrs}$.) and hospital stay was 5-21 days (mean 13 days).

The site of the tumor was in the upper outer quadrant in 28 patients, lower outer quadrant in 6 patients, lower inner quadrant in 2 patients, upper inner in one patient and central in 3 patients. Twenty-six patients had the mass in the right breast while 14 patients had left breast mass.

$28(70 \%)$ patients underwent (WLE) and reconstruction with (LD) miniflap while modified radical mastectomy, skin sparing mastectomy and subcutaneous mastectomy were done in $2(5 \%), 4(10 \%)$ and $6(15 \%)$ patients respectively with complete reconstruction by extended(LDF). Most of patients had T2 tumor size $(70 \%)$ while, T1 and T3 tumor size represented $(15 \%)$ for each. Tumors characteristics are shown in Table 1.

Table 1. Tumors Characteristics.

\begin{tabular}{lll}
\hline Tumor characteristics & Number of patients & Percentage (\%) \\
\hline Location of tumor & & \\
Upper outer quadrant & 28 & $70 \%$ \\
lower outer quadrant & 6 & $15 \%$ \\
upper inner quadrant & 1 & $2.5 \%$ \\
lower inner quadrant & 2 & $5 \%$ \\
central part & 3 & $7.5 \%$ \\
Tumor size (T) & & \\
T1 & 6 & $15 \%$ \\
T2 & 28 & $70 \%$ \\
T3 & 6 & $15 \%$ \\
Lymph Nodes & & \\
Positive & 36 & $90 \%$ \\
Negative & 4 & $10 \%$ \\
Tumor grade & & \\
Grade 1\&2 & 32 & $80 \%$ \\
Grade 3 & 8 & $20 \%$ \\
\hline
\end{tabular}

In all $\mathrm{T} 1$ (6 patients) and of T2 (22 patients) underwent conservative breast surgery by wide local excision with safety margin then underwent augmentation by LDMF as the excised volume of the breast tissue is large in comparison to the breast size that will lead to volume deformity and cosmetic appearance if we do not proceed to oncoplastic augmentation. In other patients of T2 tumor, we proceed to subcutaneous mastectomy (4 patients) or skin sparing mastectomy (2 patients) with complete reconstruction by extended LD flap because of multicentricity, positive margin in spite of repeated trial of resection or discrepancy of tumor size and breast volume.

Table 2. Type of surgery performed and complications rate in the study group.

\begin{tabular}{lll}
\hline & Number of patients & Percentage (\%) \\
\hline Type of surgery & & \\
WLE and LD miniflap & 28 & $70 \%$ \\
MRM and LD myocutaneous & 2 & $5 \%$ \\
flap & 4 & $10 \%$ \\
SSM and extended LD flap & 4 & $15 \%$ \\
SCM and extended LD flap & 6 & \\
Complication & & $10 \%$ \\
Partial flap necrosis & 4 & $5 \%$ \\
lymphorrhoea & 2 & $5 \%$ \\
wound breakdown & 2 & $15 \%$ \\
Seroma at donor sit & 6 & \\
\hline
\end{tabular}

In cases of $\mathrm{T} 3$ tumor patients had received neoadjuvant chemotherapy then the patients underwent either subcutaneous mastectomy (2 patients), skin sparing mastectomy ( 2 patients) with complete reconstruction by extended LD flap or modified radical mastectomy (2 patients that has both invasive carcinoma and large Paget's disease of the nipple and areola). Then these patients underwent coverage of the large defect left after resection using LD myocutaneous flap as shown in table 2. Tumor grade or positive axillary lymph nodes did not affect the 
decision of breast reconstruction

Neither chemotherapy nor radiotherapy were compromised or delayed in any case. Chemotherapy and radiotherapy was given without significant toxicity.

Regarding flap related complications, partial flap necrosis occurred in 4 patients $(10 \%)$, while lymphorrhoea and wound breakdown each occurred in 2 patients (5\%). Debridement and secondary suturing treated partial flap necrosis and wound breakdown. In the donor site, the most common complication was seroma, which occurred in 6 patients $(15 \%)$ and was treated by repeated aspiration in outpatient clinic as shown in table 2.

Most patients had temporary limitation of shoulder movements postoperatively but all recovered completely

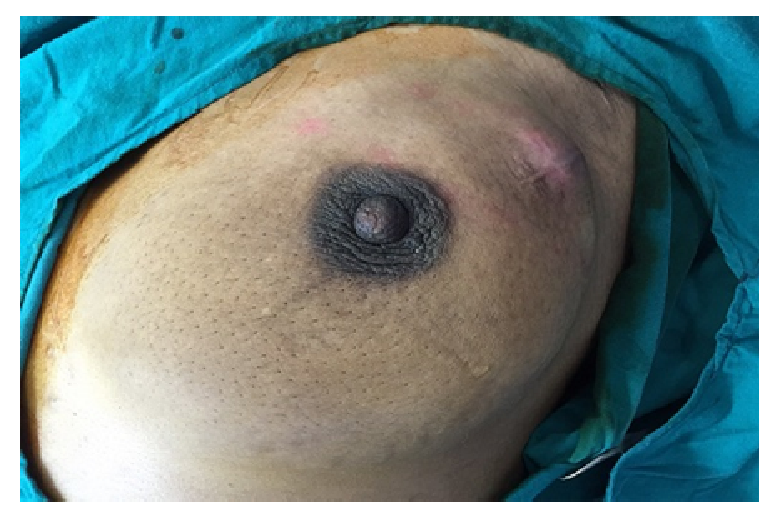

Fig. 1. Preoperative left breast cancer in upper outer quadrant.

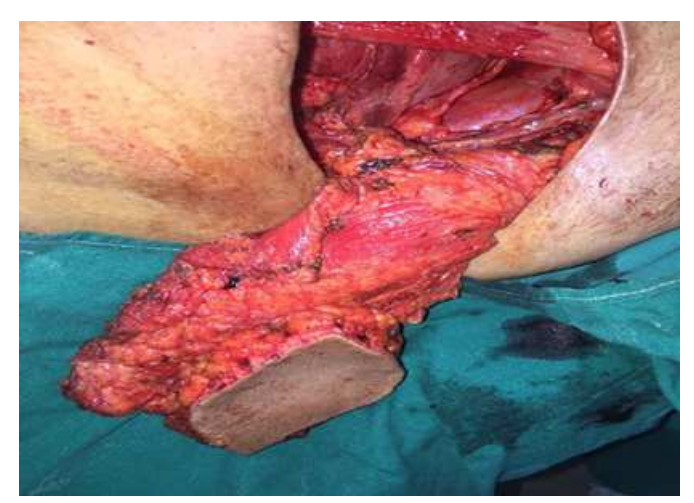

Fig. 3. Left LD flap moved to the site of partial mastectomy.

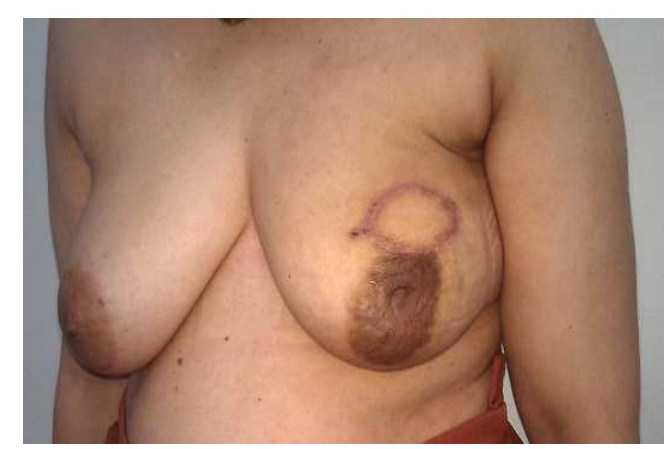

Fig. 5. Post-operative appearance after LDMF reconstruction of the left within few weeks and some of patients showed a minor deformity in the back which disappeared with time. No patients underwent secondary nipple and areola reconstruction. After follow up of 6-18 month, one patient developed contralateral breast cancer. No local recurrence or distant metastasis was recorded and all the patients were alive till the end of the study.

Evaluation of aesthetic results (Figs 1-6) by patients revealed that 30 patients (75\%) were deeply satisfied, 6 patients $(15 \%)$ were satisfied and 4 patients $(10 \%)$ were poorly satisfied. While, surgeon aesthetic evaluation was good in 28 patients $(70 \%)$, satisfactory in 8 patients $(20 \%)$ and fair in 4 patients $(10 \%)$ as shown in table 3 .

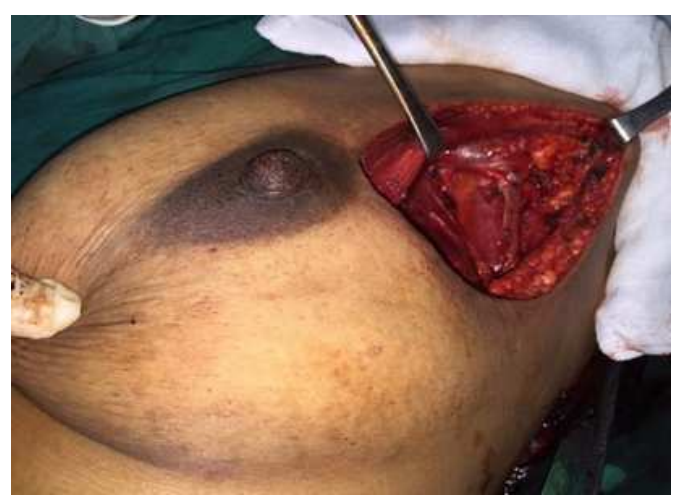

Fig. 2. Complete resection of the lesion with $2 \mathrm{~cm}$ safety margin all around and complete axillary $L N$ dissection.

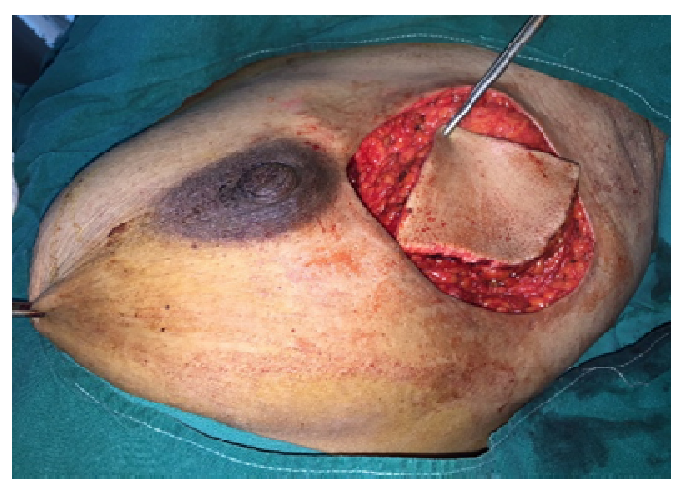

Fig. 4. Defect from partial mastectomy filled with LD flap.

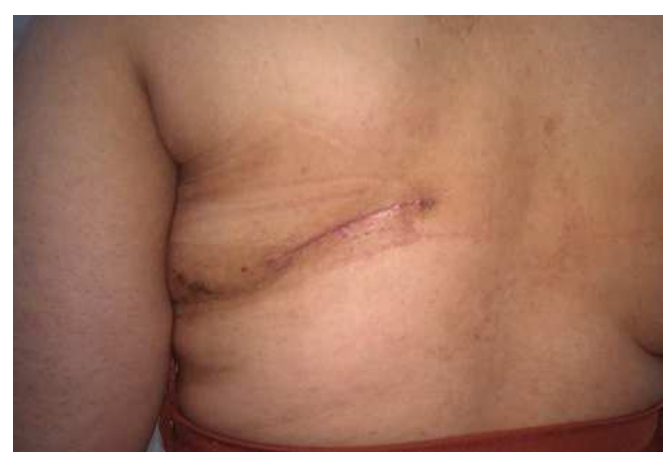

Fig. 6. Postoperative appearance of back scar. 
Table 3. Aesthetic results of LDF breast reconstruction.

\begin{tabular}{lll}
\hline Scoring by patients\& surgeons & Number of patients & Percentage \\
\hline Patients & & \\
Deeply satisfied & 30 & $75 \%$ \\
Satisfied & 6 & $15 \%$ \\
poorly satisfied & 4 & $10 \%$ \\
Surgeons & & \\
Good & 28 & $70 \%$ \\
Satisfactory & 8 & $20 \%$ \\
Fair & 4 & $10 \%$ \\
\hline
\end{tabular}

\section{Discussion}

LDF is very useful in breast reconstruction because of its well - defined vascular pedicle, safe flap elevation procedure, increased the volume of the reconstructed breast, and excellent overall results. These elements make this technique the method of choice for breast reconstruction at our clinic.

Other authors describe similar results with this technique [9]. In our series, we agree with Delay et al [10] that subtotal division of the LD tendon leaving a small muscle bridge to protect the pedicle. This may help to minimize the bulk while still offering some protection to the pedicle. This technique should lead to only a temporary bulge that improves considerably with time as it is expected that a degree of muscle disuse atrophy will occur to some extent despite keeping the thoracodorsal nerve intact.

In our study, we have designed the skin paddle in a transverse direction and we were still able to harvest enough fat from the scapular and lumbar regions. The transverse scar was quite acceptable to patients with less deformity. It is to be noted that the choice of the skin design varies from one surgeon to another. Some authors have abandoned the use of the fleur-de-lis skin paddle design because of the resulting extensive donor-site scar and have adopted to use the transverse skin paddle instead, where it can be hidden in the bra line $[11,12]$.

The LDF itself is a very reliable flap with very low incidence of partial or complete necrosis $[13,14]$. One large study quoted complete loss of the latissimus flap in one of 125 patients [15] In our study, superficial skin necrosis occurred in four patients and treated by debridement and secondary suturing without deformity. Necrosis usually happens when there is tension or twist on the pedicle. Some believe that keeping the humeral tendon insertion attached can minimize the occurrence of this problem.

The most frequent complication was donor-site seroma, with a rate (17.8\%) comparable to those published by Munhoz et al [16] and Kat t al [17], the seroma appeared after drain removal and required serial aspiration. This rate is not high if we take into account that all of the patients had undergone nodal dissection, this practice being a risk factor for seroma formation. Aiming to decrease the incidence of seroma we tried to anchor the donor site flaps to underling tissues by quilting stitches to decrease the dead space leaved after mobilization of the muscle as advocated by others [18]. Even so, this complication can be easily dealt with and does not require additional surgical procedures.

The overall patient satisfaction in our study was high as 30 patients $(75 \%)$ were deeply satisfied and $6(15 \%)$ were only satisfied, and more also the quality of Life results showed high patient satisfaction. This favorable cosmetic result therefore, justifies the use of LDF reconstruction in our practice for breast cancer surgery. The main advantage of such method is the creation of natural breast ptosis, this also mentioned by other studies [19-21].

On the other hand, the results were graded as being slightly less favorable by the surgeons due to their more critical look searching for mild asymmetry, existence of skin paddle or postoperative radiation morphologic changes on the flap. Although the tissue edema and fibrosis were more severe in the early post-irradiation period, the reconstructed breasts got softer with time and most patients were satisfied.

Patients in this study who had mild to moderate asymmetry were very reluctant to undergo simultaneous or delayed contralateral breast surgery. Similarly, Delay et al. [10] reported that the majority of their patients did not agree on contralateral breast surgery. It is well noted that completion of nipple/areolar reconstruction improves patient aesthetic satisfaction with their breast reconstructions. On the other hand, a large number of patients may just be satisfied by the newly constructed breast mound and may refuse the option of nipple and areola reconstruction [22] None of the patients whom underwent central resection in this study was willing to undergo nipple and areola reconstruction.

\section{Conclusion}

(LDF) breast reconstruction is a very versatile, safe and satisfactory technique with high success rate and is even suitable for high-risk patients. Donor site seroma is the most common complication and can be treated by repeated aspiration in outpatient clinic. (LDMF) miniflap is the mainstay of breast reconstruction after partial mastectomy to repair defects in the lateral quadrants and the lower inner pole with low donor site morbidity and deep patient satisfaction.

\section{References}

[1] Veronesi U, Cascinelli N, Mariani L, Greco M, Saccozzi R, Luini A, Aguilar M, Marubini E: Twenty-year follow-up of a randomized study comparing breast-conserving surgery with radical mastectomy for early breast cancer. $N$ Engl $J$ Med 2002, 347:1227-1232.

[2] Munhoz AM, Aldrighi CM, Ferreira MC: Paradigms in oncoplastic breast surgery: a careful assessment of the oncological need and esthetic objective. Breast $J$ 2007, 13:326-327. 
[3] Hamdi M, Wolfli J, Van Landuyt K: Partial mastectomy reconstruction. Clin Plast Surg 2007, 34:51-62; abstract vi.

[4] Munhoz AM, Montag E, Arruda E, Pellarin L, Filassi JR, Piato JR, de Barros AC, Prado LC, Fonseca A, Baracat E, Ferreira MC: Assessment of immediate conservative breast surgery reconstruction: a classification system of defects revisited and an algorithm for selecting the appropriate technique. Plast Reconstr Surg 2008, 121:716-727.

[5] Knight MA, Nguyen DTt, Kobayashi MR, Evans GR: Institutional review of free TRAM flap breast reconstruction. Ann Plast Surg 2006, 56:593-598.

[6] Eriksen C, Stark B: The latissimus dorsi flap--still a valuable tool in breast reconstruction: report of 32 cases. Scand J Plast Reconstr Surg Hand Surg 2008, 42:132-137.

[7] Noguchi M, Taniya T, Miyazaki I, Saito Y: Immediate transposition of a latissimus dorsi muscle for correcting a postquadrantectomy breast deformity in Japanese patients. Int Surg 1990, 75:166-170.

[8] Raja MA, Straker VF, Rainsbury RM: Extending the role of breast-conserving surgery by immediate volume replacement. Br J Surg 1997, 84:101-105.

[9] Chang DW, Barnea Y, Robb GL: Effects of an autologous flap combined with an implant for breast reconstruction: an evaluation of 1000 consecutive reconstructions of previously irradiated breasts. Plast Reconstr Surg 2008, 122:356-362.

[10] Delay E, Gounot N, Bouillot A, Zlatoff P, Rivoire M: Autologous latissimus breast reconstruction: a 3-year clinical experience with 100 patients. Plast Reconstr Surg 1998, 102:1461-1478.

[11] Chang DW, Youssef A, Cha S, Reece GP: Autologous breast reconstruction with the extended latissimus dorsi flap. Plast Reconstr Surg 2002, 110:751-759; discussion 760-751.

[12] Menke H, Erkens M, Olbrisch RR: Evolving concepts in breast reconstruction with latissimus dorsi flaps: results and follow-up of 121 consecutive patients. Ann Plast Surg 2001, 47:107-114

[13] Barnett GR, Gianoutsos MP: The latissimus dorsi added fat flap for natural tissue breast reconstruction: report of 15 cases. Plast Reconstr Surg 1996, 97:63-70.

[14] Roy MK, Shrotia S, Holcombe C, Webster DJ, Hughes LE, Mansel RE: Complications of latissimus dorsi myocutaneous flap breast reconstruction. Eur J Surg Oncol 1998, 24:162-165.

[15] Papp C, McCraw JB: Autogenous latissimus breast reconstruction. Clin Plast Surg 1998, 25:261-266.

[16] Munhoz AM, Montag E, Fels KW, Arruda EG, Sturtz GP, Aldrighi C, Gemperli R, Ferreira MC: Outcome analysis of breast-conservation surgery and immediate latissimus dorsi flap reconstruction in patients with $\mathrm{T} 1$ to $\mathrm{T} 2$ breast cancer. Plast Reconstr Surg 2005, 116:741-752.

[17] Kat CC, Darcy CM, O'Donoghue JM, Taylor AR, Regan PJ: The use of the latissimus dorsi musculocutaneous flap for immediate correction of the deformity resulting from breast conservation surgery. Br J Plast Surg 1999, 52:99-103.

[18] Titley OG, Spyrou GE, Fatah MF: Preventing seroma in the latissimus dorsi flap donor site. Br J Plast Surg 1997, 50:106108.

[19] Gendy RK, Able JA, Rainsbury RM: Impact of skin-sparing mastectomy with immediate reconstruction and breast-sparing reconstruction with miniflaps on the outcomes of oncoplastic breast surgery. Br J Surg 2003, 90:433-439.

[20] Navin C, Agrawal A, Kolar KM: The use of latissimus dorsi miniflap for reconstruction following breast-conserving surgery: experience of a small breast unit in a district hospital. World J Surg 2007, 31:46-50.

[21] Rifaat MA, Amin AA, Bassiouny M, Nabawi A, Monib S: The extended latissimus dorsi flap option in autologous breast reconstruction: A report of 14 cases and review of the literature. Indian J Plast Surg 2008, 41:24-33.

[22] Randolph LC, Barone J, Angelats J, Dado DV, Vandevender DK, Shoup M: Prediction of postoperative seroma after latissimus dorsi breast reconstruction. Plast Reconstr Surg 2005, 116:1287-1290. 